\title{
Spherically symmetric model atmospheres using approximate lambda operators
}

\section{Computational details of the thermal balance method}

\author{
J. Kubát \\ Astronomický ústav, Akademie věd České republiky, CZ-251 65 Ondřejov, Czech Republic \\ Received 11 September 2000 / Accepted 14 November 2000

\begin{abstract}
The condition of a thermal balance of electrons is used in a linearization method of calculation of of the method are presented and discussed. The method is shown to be robust and powerful. It is superior to the commonly used method based on the condition of radiative equilibrium.
\end{abstract} \\ spherically symmetric NLTE model atmospheres in hydrostatic and radiative equilibrium. Computational details
}

Key words. stars: atmospheres - methods: numerical - radiative transfer

\section{Introduction}

In our previous papers (Kubát 1994 - hereinafter Paper I; Kubát 1996 - hereinafter Paper II; Kubát 1997c - hereinafter Paper III) we have presented a description of a method for the calculation of static spherically symmetric NLTE model atmospheres in radiative and hydrostatic equilibrium. The code based on this method was applied to checking the sphericity effects in a various number of stars (e.g. Kubát 1995, 1997a, 1999) and to the study of the effects of different $\mathrm{H} / \mathrm{He}$ ratios in hot white dwarfs (Kubát 1997b).

In Kubát et al. (1999 - hereinafter KPP) we presented an extremely powerful method for calculation of temperature in the outer layers of NLTE model atmospheres. Our method is based on the equation of thermal balance of electrons and it was shown that this method is superior to the standard method using the equation of radiative equilibrium. Our method as described in Papers I to III and in KPP is suitable for calculation of model atmospheres of hot stars that are convectively stable.

In this paper, we present numerical details of the method presented in KPP and we apply our method to calculations of static spherically symmetric NLTE model atmospheres.

Send offprint requests to: J. Kubát, e-mail: kubat@sunstel.asu.cas.cz

\section{Energy equilibrium in stellar atmospheres}

\subsection{Radiative equilibrium}

The temperature structure in stellar atmospheres is usually determined using some condition of conservation of energy or of an energy equilibrium. Let us assume that energy is transported through the stellar atmosphere only via radiation, i.e., we neglect convection. Therefore, we restrict our calculations to hot stars, where atmospheric convection is not important. The standard equation is the equation of radiative equilibrium (see, e.g. Mihalas 1978), which simply states that the total amount of radiative energy absorbed in a particular region of a stellar atmosphere is again reemitted in the same region. In other words, the total radiative flux is conserved. Mathematical expressions for these two statements differ a bit. The former one is the so-called integral equation of radiative equilibrium

$4 \pi \int_{0}^{\infty}\left(\kappa_{\nu} J_{\nu}-\eta_{\nu}\right) \mathrm{d} \nu=0$

whereas the latter one is the so-called differential equation of radiative equilibrium

$\nabla \cdot \mathcal{F}=0$.

The Eq. (2) ensures the flux conservation, but it is numerically unstable for low optical depths. On the other hand, Eq. (1) is stable but it does not guarantee the flux conservation at large optical depths. Therefore it is convenient 
to use Eq. (1) in the outer parts and Eq. (2) in the inner parts of the atmosphere. Both forms have been discussed in detail in Paper II.

\subsection{Thermal balance of electrons}

An alternative to the Eq. (1) was suggested by KPP. It is based on a method introduced by Hummer \& Seaton (1963) and Hummer (1963), which was then commonly used in the studies of planetary nebulae (e.g. Williams 1967; Ferland \& Truran 1981). The method was also discussed by Hoffmann (1995) and Škoda (1995). Only several applications to calculations of NLTE model atmospheres appeared (Drew 1985, 1989; Pauldrach et al. 1997; Hillier \& Miller 1998). It is possible to use the equation of thermal balance of electrons which considers heating and cooling of an electron gas by collisions with atoms, by radiative ionization and recombination, and via free-free transitions. All these processes may be summarized in the following equation of thermal balance of electrons

$\left(Q_{\mathrm{c}}^{\mathrm{H}}+Q_{\mathrm{bf}}^{\mathrm{H}}+Q_{\mathrm{ff}}^{\mathrm{H}}\right)-\left(Q_{\mathrm{c}}^{\mathrm{C}}+Q_{\mathrm{bf}}^{\mathrm{C}}+Q_{\mathrm{ff}}^{\mathrm{C}}\right)=0$.

Here, $Q_{\mathrm{c}}^{\mathrm{H}}$ is the heating of the electron gas by collisions with atoms (this term corresponds to the collisional deexcitation and recombination), $Q_{\mathrm{c}}^{\mathrm{C}}$ is the cooling of the electron gas by collisions (corresponding to collisional excitation and ionization), $Q_{\mathrm{bf}}^{\mathrm{H}}$ is the heating caused by a part of a photon energy not absorbed in photoionization, $Q_{\mathrm{bf}}^{\mathrm{C}}$ is the cooling caused by photorecombination, $Q_{\mathrm{ff}}^{\mathrm{H}}$ is the heating by free-free absorption, and finally $Q_{\mathrm{ff}}^{\mathrm{C}}$ is the free-free cooling. Expressions for these quantities can be found in KPP, Eqs. (3-12). This method was shown to be powerful and more stable than the standard Eq. (1). A detailed discussion can be found in KPP.

\section{Computational details}

In order to obtain the atmospheric structure we have to solve several additional equations to the temperature equation (the equation of thermal balance), namely the equation of hydrostatic equilibrium, the equations of statistical equilibrium, and the radiative transfer equation. We shall not repeat the discussion of the above mentioned equations that appeared in Papers I, II, and III, here we shall only discuss some interesting details which we regard to be important.

\subsection{Determination of temperature}

There are two options how to calculate the temperature structure of the outer parts of the model atmosphere, namely the equation of radiative equilibrium and the equation of thermal balance of electrons, as described in Sect. 2. The temperature at the inner parts is successfully determined using the differential form of the equation of radiative equilibrium (Eq. (2)).

During the last two years we have calculated a large number of NLTE model atmospheres using both the radiative equilibrium and thermal balance methods. We can summarize our computational experience as follows. The thermal balance method is faster and more efficient for most of the models we have calculated (early B and hotter, hot white dwarfs). The only problems came out when we turned to cooler stars as late B and early A. For these models the thermal balance method is numerically unstable at few depth points "in the middle" of the atmosphere, mostly near the point where the temperature of the NLTE model starts to rise with increasing radius. This difficulty can be easily overcome by using the equation of radiative equilibrium for these few points.

\subsection{Radiative transfer}

The radiative transfer equation in the linearization step is treated with the help of approximate lambda operators, i.e. the mean intensity of radiation is expressed as

$J_{\nu}=\Lambda_{\nu}^{*} S_{\nu}+\left(\Lambda_{\nu}-\Lambda_{\nu}^{*}\right) S_{\nu}=\Lambda_{\nu}^{*} S_{\nu}+\Delta J_{\nu}$.

The approximate diagonal or tridiagonal lambda operator $\Lambda_{\nu}^{*}$ is calculated after the formal solution of the transfer equation following the idea of Rybicki \& Hummer (1991) and Puls (1991). This ensures that the lambda operator is numerically consistent with the formal solution of the radiative transfer equation. Although tridiagonal operators were introduced into model atmosphere calculation by Werner (1989) in order to make the calculations faster, our experience shows that it is more useful to use a simpler form of a diagonal operator. Calculations in each iteration step are much faster and the benefit of more rapid convergence of the tridiagonal operator is not significant.

The formal solution of the transfer equation in spherical geometry is calculated using either 2nd order differences (Mihalas 1985), splines, or Hermite solution (Auer 1976) using Feautrier (1964) variables. More details about the radiative transfer in our code can be found in Papers I and II.

\subsection{Linearization of model atmosphere equations}

The resulting system of equations (hydrostatic equilibrium, thermal balance, radiative equilibrium, radiative transfer, statistical equilibrium) is discretized and solved using the Newton-Raphson method, which has been usually called the complete linearization method since its introduction to model atmosphere calculations by Auer \& Mihalas (1969). The essence of this method is in iterative calculation of corrections to the vector of variables $\boldsymbol{\psi}=\left(T, n_{\mathrm{e}}, b_{l}\right)$, where $T$ is temperature, $n_{\mathrm{e}}$ is the electron density, and $b_{l}$ are the departure coefficients of particular atomic levels. The iterations are repeated until convergence is reached. The model is said to be converged if the relative corrections $\delta \psi / \psi$ are less than some value. Our experience shows that the value of $10^{-4}$ gives satisfactory results. 
We would like to obtain (after some discretization and linearization) a tridiagonal system of the standard form

$$
\mathbf{A}_{d} \delta \boldsymbol{\psi}_{d-1}+\mathbf{B}_{d} \delta \boldsymbol{\psi}_{d}+\mathbf{C}_{d} \delta \boldsymbol{\psi}_{d+1}=\boldsymbol{L}_{d}, \quad d=1 \ldots \mathrm{ND}
$$

$\mathbf{A}_{1}=\mathbf{C}_{\mathrm{ND}}=0$ ( $\mathrm{ND}$ is the total number of depth points). This system can be solved by standard Gaussian elimination (see Paper I).

\subsection{Thermal balance}

Discretization and linearization of equations of hydrostatic, radiative, and statistical equilibrium were described in detail in Papers I, II, and III. Here we concentrate on the description of handling the thermal balance equation.

We aim at writing the discretized and linearized equation of thermal balance in the form

$$
\begin{aligned}
& \left(\mathbf{A}_{T}^{E E}\right)_{d}(\delta T)_{d-1}+\left(\mathbf{A}_{n_{\mathrm{e}}}^{E E}\right)_{d}\left(\delta n_{\mathrm{e}}\right)_{d-1} \\
& +\left(\mathbf{A}_{b_{l}}^{E E}\right)_{d}\left(\delta b_{l}\right)_{d-1}+\left(\mathbf{B}_{T}^{E E}\right)_{d}(\delta T)_{d}+\left(\mathbf{B}_{n_{\mathrm{e}}}^{E E}\right)_{d}\left(\delta n_{\mathrm{e}}\right)_{d} \\
& +\left(\mathbf{B}_{b_{l}}^{E E}\right)_{d}\left(\delta b_{l}\right)_{d}+\left(\mathbf{C}_{T}^{E E}\right)_{d}(\delta T)_{d+1}+\left(\mathbf{C}_{n_{\mathrm{e}}}^{E E}\right)_{d}\left(\delta n_{\mathrm{e}}\right)_{d+1} \\
& +\left(\mathbf{C}_{b_{l}}^{E E}\right)_{d}\left(\delta b_{l}\right)_{d+1}=\boldsymbol{L}_{d}^{E E}
\end{aligned}
$$

Details of this cumbersome procedure are presented in Appendix A. The Eq. (6) is used at each depth point. In addition, at large optical depths $\left(\tau_{\mathrm{R}} \gtrsim 2 / 3\right)$, where it is necessary to guarantee the flux conservation, the latter equation is superposed with the differential form of radiative equilibrium (2) (see Hubeny \& Lanz 1995 and Paper II).

\subsection{Acceleration of convergence}

It is very advantageous to use some technique to accelerate convergence of model atmosphere calculations. We have implemented two basic methods, namely the $\mathrm{Ng}$ and Kantorovich accelerations.

The Kantorovich acceleration (see, e.g., Kantorovich \& Akilov 1977) was brought into NLTE model atmosphere calculations by Hubeny \& Lanz (1992). It is based on a fact that near the converged solution the Jacobians of the Newton-Raphson method in subsequent iterations differ only slightly and that this difference has very little influence on the convergence properties. The idea is not to calculate all these Jacobians and to replace them by a value found in one of the preceding iterations. Coding is very simple and the saving of the compiler time is considerable. In fact it is not a real acceleration of convergence in a numerical sense (it does not lower the number of iterations), it is rather a method of huge saving of the computer time.

A second technique is the so-called $\mathrm{Ng}$ acceleration. It was described by $\mathrm{Ng}$ (1974) and then first applied to radiative transfer by Olson et al. (1986). This technique calculates new solution of $\boldsymbol{\psi}$ using information not only from previous iteration step, but also from earlier ones. It accelerates the convergence significantly, but sometimes it may lead to overestimates and divergence. A very useful description of this method was written by Auer (1987).

We used the acceleration techniques in our inner iteration cycle (see Fig. 1 in Paper I). Similarly to Rybicki \& Hummer (1991), our experience shows that it is useful to switch the accelerations on after several $(\sim 7)$ normal (i.e. without acceleration) iterations. We found that using of the Kantorovich acceleration always helps, whereas Ng's acceleration must be used with care, since it sometimes leads to divergence. A useful method to overcome the difficulties with $\mathrm{Ng}$ acceleration is not to allow it until the relative changes drop below $10^{-2}$.

\section{Conclusions}

We have implemented an important technique for the determination of temperature in stellar atmospheres into our static spherically symmetric code. This technique is the determination of the temperature structure in the outer layers using the method of thermal balance of electrons. It was shown to be more powerful and more convenient than the standard method of determination of temperature using the equation of radiative equilibrium.

Using this technique, we calculated a number of spherically symmetric NLTE model atmospheres in hydrostatic and radiative equilibrium. Detailed results of our calculations will be published elsewhere.

Acknowledgements. The author would like to thank Dr. Frédéric Paletou for a careful reading of the manuscript and for valuable comments. This work was supported by a grant of the Grant Agency of the Czech Republic (GA ČR) 205/96/1198, by an internal grant of Academy of Sciences of the Czech Republic C3003805, and by projects K1-003-601/4 and K1-043-601.

\section{Appendix A: Details of linearization of the electron thermal balance equation}

First, we shall derive discretized equations for net heating rates. In the following $d(d=1, \ldots, N D)$ is the depth index and $i$ is the frequency index. The equations from KPP are denoted as (KPP.x) The expression for each net free-free heating follows from Eqs. (KPP.3) and (KPP.4), namely

$\left(Q_{\mathrm{ff}}\right)_{d}=\left(K_{\mathrm{ff}}\right)_{d} \sum_{i=1}^{N F} w_{i}\left(\alpha_{\mathrm{ff}}\right)_{i}\left(T_{d}\right) Y_{d i}$

where

$\left(K_{\mathrm{ff}}\right)_{d}=4 \pi\left(n_{\mathrm{e}}\right)_{d}\left(b_{k}\right)_{d}\left(n_{k}^{*}\right)_{d}$,

$Y_{d i}=\tilde{\mathcal{J}}_{d i}-\tilde{\mathcal{E}}_{d i}$

$\tilde{\mathcal{J}}_{d, i}=\Lambda_{d, d-1, i}^{*} S_{d-1, i}+\Lambda_{d, d, i}^{*} S_{d, i}$ $+\Lambda_{d, d+1, i}^{*} S_{d+1, i}+\Delta J_{d, i}$

and

$\tilde{\mathcal{E}}_{d i}=\left(\tilde{\mathcal{J}}_{d i}+\frac{2 h \nu_{i}^{3}}{c^{2}}\right) \mathrm{e}^{-h \nu_{i} / k T_{d}}$. 
Similarly, we obtain the discretized expression for each net bound-free heating from Eqs. (KPP.5) and (KPP.6)

$$
\begin{aligned}
\left(Q_{\mathrm{bf}}\right)_{d}= & \left(K_{\mathrm{bf}}\right)_{d} \sum_{i=1}^{N F} w_{i}\left(\alpha_{\mathrm{bf}}\right)_{i} \tilde{\mathcal{J}}_{d i} \\
& -\left(K_{\mathrm{fb}}\right)_{d} \sum_{i=1}^{N F} w_{i}\left(\alpha_{\mathrm{bf}}\right)_{i} \tilde{\mathcal{E}}_{d i}
\end{aligned}
$$

where

$\left(K_{\mathrm{bf}}\right)_{d}=4 \pi\left(n_{l}^{*}\right)_{d}\left(b_{l}\right)_{d}\left(w_{k}\right)_{d}$,

$$
\left(K_{\mathrm{fb}}\right)_{d}=4 \pi\left(n_{l}^{*}\right)_{d}\left(b_{k}\right)_{d}\left(w_{l}\right)_{d}
$$

and for net collisional heating from Eqs. (KPP.11) and (KPP.12)

$$
\begin{aligned}
\left.\frac{\partial\left(Q_{\mathrm{ff}}\right)_{d}}{\partial T}\right|_{d}= & \left.\frac{1}{\left(n_{k}^{*}\right)_{d}} \frac{\partial n_{k}^{*}}{\partial T}\right|_{d}\left(Q_{\mathrm{ff}}\right)_{d} \\
& +\left(K_{\mathrm{ff}}\right)_{d}\left(\left.\sum_{i=1}^{N F} w_{i}\left(\alpha_{\mathrm{ff}}\right)_{i}\left(T_{d}\right) \frac{\partial Y_{d i}}{\partial T}\right|_{d}\right. \\
& +\left.\sum_{i=1}^{N F} w_{i} \frac{\partial\left(\alpha_{\mathrm{ff}}\right)_{i}(T)}{\partial T}\right|_{d} Y_{d i} \\
& \left.-\frac{h}{k T^{2}} \sum_{i=1}^{N F} w_{i}\left(\alpha_{\mathrm{ff}}\right)_{i}\left(T_{d}\right) \tilde{\mathcal{E}}_{d i}\right)
\end{aligned}
$$

$$
\left.\frac{\partial\left(Q_{\mathrm{ff}}\right)_{d}}{\partial b_{l}}\right|_{d}=\frac{\left(Q_{\mathrm{ff}}\right)_{d}}{\left(b_{l}\right)_{d}}+\left.\left(K_{\mathrm{ff}}\right)_{d} \sum_{i=1}^{N F} w_{i}\left(\alpha_{\mathrm{ff}}\right)_{i}\left(T_{d}\right) \frac{\partial Y_{d i}}{\partial b_{l}}\right|_{d}
$$

$$
\begin{aligned}
\left(Q_{\mathrm{c}}\right)_{d}= & {\left[\left(b_{m}\right)_{d}-\left(b_{l}\right)_{d}\right] } \\
& \times\left(n_{l}^{*}\right)_{d}\left(w_{m}\right)_{d}\left(n_{\mathrm{e}}\right)_{d} q_{l m}\left(T_{d}\right) h \nu_{l m} .
\end{aligned}
$$

\section{A.1. Linearization coefficients}

After linearization we arrive at the particular coefficients for temperature and electron density of the form $(x$ stands for $n_{\mathrm{e}}, T$, or $\left.b_{l}\right)$

$$
\begin{aligned}
\left(\mathbf{A}_{x}^{E E}\right)_{d}= & \left.\sum_{\mathrm{ff}} \frac{\partial\left(Q_{\mathrm{ff}}\right)_{d}}{\partial x}\right|_{d-1}+\left.\sum_{\mathrm{bf}} \frac{\partial\left(Q_{\mathrm{bf}}\right)_{d}}{\partial x}\right|_{d-1} \\
& +\left.\sum_{\mathrm{c}} \frac{\partial\left(Q_{\mathrm{c}}\right)_{d}}{\partial x}\right|_{d-1} \\
\left(\mathbf{B}_{x}^{E E}\right)_{d}= & \left.\sum_{\mathrm{ff}} \frac{\partial\left(Q_{\mathrm{ff}}\right)_{d}}{\partial x}\right|_{d}+\left.\sum_{\mathrm{bf}} \frac{\partial\left(Q_{\mathrm{bf}}\right)_{d}}{\partial x}\right|_{d} \\
& +\left.\sum_{\mathrm{c}} \frac{\partial\left(Q_{\mathrm{c}}\right)_{d}}{\partial x}\right|_{d} \\
\left(\mathbf{C}_{x}^{E E}\right)_{d}= & \left.\sum_{\mathrm{ff}} \frac{\partial\left(Q_{\mathrm{ff}}\right)_{d}}{\partial x}\right|_{d+1}+\left.\sum_{\mathrm{bf}} \frac{\partial\left(Q_{\mathrm{bf}}\right)_{d}}{\partial x}\right|_{d+1} \\
& +\left.\sum_{\mathrm{c}} \frac{\partial\left(Q_{\mathrm{c}}\right)_{d}}{\partial x}\right|_{d+1}
\end{aligned}
$$

where $\sum_{\mathrm{ff}}, \sum_{\mathrm{bf}}$, and $\sum_{\mathrm{c}}$ mean sums over all free-free, bound-free, and collisional transitions, respectively.

\section{A.1.1. Free-free transitions}

$\left.\frac{\partial\left(Q_{\mathrm{ff}}\right)_{d}}{\partial x}\right|_{d-1}=\left.\left(K_{\mathrm{ff}}\right)_{d} \sum_{i=1}^{N F} w_{i}\left(\alpha_{\mathrm{ff}}\right)_{i}\left(T_{d}\right) \frac{\partial Y_{d i}}{\partial x}\right|_{d-1}$

$$
\left.\frac{\partial\left(Q_{\mathrm{ff}}\right)_{d}}{\partial x}\right|_{d+1}=\left.\left(K_{\mathrm{ff}}\right)_{d} \sum_{i=1}^{N F} w_{i}\left(\alpha_{\mathrm{ff}}\right)_{i}\left(T_{d}\right) \frac{\partial Y_{d i}}{\partial x}\right|_{d+1}
$$

$$
\begin{aligned}
\left.\frac{\partial\left(Q_{\mathrm{ff}}\right)_{d}}{\partial n_{\mathrm{e}}}\right|_{d}= & \frac{\left(Q_{\mathrm{ff}}\right)_{d}}{\left(n_{\mathrm{e}}\right)_{d}}+\left.\frac{1}{\left(n_{k}^{*}\right)_{d}} \frac{\partial n_{k}^{*}}{\partial n_{\mathrm{e}}}\right|_{d}\left(Q_{\mathrm{ff}}\right)_{d} \\
& +\left.\left(K_{\mathrm{ff}}\right)_{d} \sum_{i=1}^{N F} w_{i}\left(\alpha_{\mathrm{ff}}\right)_{i}\left(T_{d}\right) \frac{\partial Y_{d i}}{\partial n_{\mathrm{e}}}\right|_{d}
\end{aligned}
$$

where $(D=d-1, d$, or $d+1)$

$$
\left.\frac{\partial Y_{d i}}{\partial x}\right|_{D}=\left.\Lambda_{d D i}^{*} \frac{\partial S_{i}}{\partial x}\right|_{D}\left(1-\mathrm{e}^{-h \nu_{i} / k T_{d}}\right)
$$

\section{A.1.2. Bound-free transitions}

In this section, $x$ stands only for $n_{\mathrm{e}}$ or $T$, and

$$
\left.\frac{\partial Y_{d i}^{a}}{\partial x}\right|_{D}=\left.\Lambda_{d D i}^{*} \frac{\partial S_{i}}{\partial x}\right|_{D}
$$

$\left.\frac{\partial Y_{d i}^{e}}{\partial x}\right|_{D}=\left.\Lambda_{d D i}^{*} \frac{\partial S_{i}}{\partial x}\right|_{D} \mathrm{e}^{-h \nu_{i} / k T_{d}}$

$(D=d-1, d$, or $d+1)$

$$
\begin{aligned}
\left.\frac{\partial\left(Q_{\mathrm{bf}}\right)_{d}}{\partial x}\right|_{d-1}= & \left.\left(K_{\mathrm{bf}}\right)_{d} \sum_{i=1}^{N F} w_{i}\left(\alpha_{\mathrm{bf}}\right)_{i} \frac{\partial Y_{d i}^{a}}{\partial x}\right|_{d-1} \\
& -\left.\left(K_{\mathrm{fb}}\right)_{d} \sum_{i=1}^{N F} w_{i}\left(\alpha_{\mathrm{bf}}\right)_{i} \frac{\partial Y_{d i}^{e}}{\partial x}\right|_{d-1}
\end{aligned}
$$

$$
\begin{aligned}
\left.\frac{\partial\left(Q_{\mathrm{bf}}\right)_{d}}{\partial x}\right|_{d+1}= & \left.\left(K_{\mathrm{bf}}\right)_{d} \sum_{i=1}^{N F} w_{i}\left(\alpha_{\mathrm{bf}}\right)_{i} \frac{\partial Y_{d i}^{a}}{\partial x}\right|_{d+1} \\
& -\left.\left(K_{\mathrm{fb}}\right)_{d} \sum_{i=1}^{N F} w_{i}\left(\alpha_{\mathrm{bf}}\right)_{i} \frac{\partial Y_{d i}^{e}}{\partial x}\right|_{d+1}
\end{aligned}
$$




$$
\begin{aligned}
\left.\frac{\partial\left(Q_{\mathrm{bf}}\right)_{d}}{\partial x}\right|_{d}= & \left.\frac{1}{\left(n_{k}^{*}\right)_{d}} \frac{\partial n_{k}^{*}}{\partial x}\right|_{d}\left(Q_{\mathrm{bf}}\right)_{d} \\
& +\left.\left(K_{\mathrm{bf}}\right)_{d} \sum_{i=1}^{N F} w_{i}\left(\alpha_{\mathrm{bf}}\right)_{i} \frac{\partial Y_{d i}^{a}}{\partial x}\right|_{d} \\
& -\left.\left(K_{\mathrm{fb}}\right)_{d} \sum_{i=1}^{N F} w_{i}\left(\alpha_{\mathrm{bf}}\right)_{i} \frac{\partial Y_{d i}^{e}}{\partial x}\right|_{d} \\
& -\delta_{T x}\left(K_{\mathrm{fb}}\right)_{d} \frac{h}{k T^{2}} \sum_{i=1}^{N F} w_{i}\left(\alpha_{\mathrm{bf}}\right)_{i} \tilde{\mathcal{E}}_{d i}
\end{aligned}
$$

where $\delta_{T x}=1$ for $x=T$, otherwise it is 0 .

$$
\begin{aligned}
& \left.\frac{\partial\left(Q_{\mathrm{bf}}\right)_{d}}{\partial b_{l}}\right|_{d-1}=\left.\left(K_{\mathrm{bf}}\right)_{d} \sum_{i=1}^{N F} w_{i}\left(\alpha_{\mathrm{bf}}\right)_{i} \frac{\partial Y_{d i}^{a}}{\partial b_{l}}\right|_{d-1} \\
& \left.\frac{\partial\left(Q_{\mathrm{bf}}\right)_{d}}{\partial b_{l}}\right|_{d}=\left(K_{\mathrm{bf}}\right)_{d} \sum_{i=1}^{N F} w_{i}\left(\alpha_{\mathrm{bf}}\right)_{i}\left(\frac{\mathcal{J}_{d i}}{b_{l}}+\left.\frac{\partial Y_{d i}^{a}}{\partial b_{l}}\right|_{d}\right) \\
& \left.\frac{\partial\left(Q_{\mathrm{bf}}\right)_{d}}{\partial b_{l}}\right|_{d+1}=\left.\left(K_{\mathrm{bf}}\right)_{d} \sum_{i=1}^{N F} w_{i}\left(\alpha_{\mathrm{bf}}\right)_{i} \frac{\partial Y_{d i}^{a}}{\partial b_{l}}\right|_{d+1} \\
& \left.\frac{\partial\left(Q_{\mathrm{bf}}\right)_{d}}{\partial b_{k}}\right|_{d-1}=-\left.\left(K_{\mathrm{bf}}\right)_{d} \sum_{i=1}^{N F} w_{i}\left(\alpha_{\mathrm{bf}}\right)_{i} \frac{\partial Y_{d i}^{e}}{\partial b_{k}}\right|_{d-1} \\
& \left.\frac{\partial\left(Q_{\mathrm{bf}}\right)_{d}}{\partial b_{k}}\right|_{d}=-\left(K_{\mathrm{bf}}\right)_{d} \sum_{i=1}^{N F} w_{i}\left(\alpha_{\mathrm{bf}}\right)_{i}\left(\frac{\mathcal{E}_{d i}}{b_{k}}+\left.\frac{\partial Y_{d i}^{e}}{\partial b_{k}}\right|_{d}\right)_{d}(\mathrm{~A} \\
& \left.\frac{\partial\left(Q_{\mathrm{bf}}\right)_{d}}{\partial b_{k}}\right|_{d+1}=-\left.\left(K_{\mathrm{bf}}\right)_{d} \sum_{i=1}^{N F} w_{i}\left(\alpha_{\mathrm{bf}}\right)_{i} \frac{\partial Y_{d i}^{e}}{\partial b_{k}}\right|_{d+1}
\end{aligned}
$$

\section{A.1.3. Collisions}

$$
\begin{aligned}
\left.\frac{\partial\left(Q_{\mathrm{c}}\right)_{d}}{\partial n_{\mathrm{e}}}\right|_{d}= & \left(\frac{1}{\left(n_{\mathrm{e}}\right)_{d}}+\left.\frac{1}{\left(n_{l}^{*}\right)_{d}} \frac{\partial n_{l}^{*}}{\partial n_{\mathrm{e}}}\right|_{d}\right)\left(Q_{\mathrm{c}}\right)_{d} \\
\left.\frac{\partial\left(Q_{\mathrm{c}}\right)_{d}}{\partial T}\right|_{d}= & \left(\left.\frac{1}{q_{l m}\left(T_{d}\right)} \frac{\partial q_{l m}(T)}{\partial T}\right|_{d}+\left.\frac{1}{\left(n_{l}^{*}\right)_{d}} \frac{\partial n_{l}^{*}}{\partial T}\right|_{d}\right) \\
& \times\left(Q_{\mathrm{c}}\right)_{d}
\end{aligned}
$$

$$
\begin{aligned}
& \left(Q_{\mathrm{c}}\right)_{d}=-\left(n_{l}^{*}\right)_{d}\left(w_{m}\right)_{d}\left(n_{\mathrm{e}}\right)_{d} q_{l m}\left(T_{d}\right) h \nu_{l m} \\
& \left(Q_{\mathrm{c}}\right)_{d}=\left(n_{l}^{*}\right)_{d}\left(w_{m}\right)_{d}\left(n_{\mathrm{e}}\right)_{d} q_{l m}\left(T_{d}\right) h \nu_{l m} .
\end{aligned}
$$

\section{References}

Auer, L. H. 1976, JQSRT, 16, 931

Auer, L. H. 1987, in Numerical Radiative Transfer, ed., W. Kalkofen (Cambridge Univ. Press), 101

Auer, L. H., \& Mihalas, D. 1969, ApJ, 158, 641

Drew, J. 1985, MNRAS, 217, 867

Drew, J. 1989, ApJS, 71, 267

Feautrier, P. 1964, C. R. Acad. Sci. Paris, 258, 3189

Ferland, G. J., \& Truran, J. W. 1981, ApJ, 244, 1022

Hillier, D. J., \& Miller, D. L. 1998, ApJ, 496, 407

Hoffmann, T. L. 1995, Diplomarbeit, Universität München

Hubeny, I., \& Lanz, T. 1992, A\&A, 262, 501

Hubeny, I., \& Lanz, T. 1995, ApJ, 439, 875

Hummer, D. G. 1963, MNRAS, 125, 461

Hummer, D. G., \& Seaton, M. J. 1963, MNRAS, 125, 437

Kantorovich, L. V., \& Akilov, G. P. 1977, Funkcionalyj analiz, 2nd ed. (Nauka, Moskva)

Kubát, J. 1994, A\&A, 287, 179, Paper I

Kubát, J. 1995, A\&A, 299, 803

Kubát, J. 1996, A\&A, 305, 255, Paper II

Kubát, J. 1997a, A\&A, 323, 524

Kubát, J. 1997b, A\&A, 324, 1020

Kubát, J. 1997c, A\&A, 326, 277, Paper III

Kubát, J. 1999, NewA, 4, 157

Kubát, J., Puls, J., \& Pauldrach, A. W. A. 1999, A\&A, 341, $587(\mathrm{KPP})$

Mihalas, D. 1978, Stellar Atmospheres, 2nd ed. (W. H. Freeman, \& Comp., San Francisco)

Mihalas, D. 1985, J. Comput. Phys., 57, 1

Ng, K. C. 1974, J. Chem. Phys., 61, 2680

Olson, G. L., Auer, L. H., \& Buchler, J. R. 1986, JQSRT, 35, 431

Pauldrach, A. W. A., Lennon, M., Hoffmann, T. L., et al. 1997, in Properties of Hot Luminous Stars, Boulder-Munich Workshop II, ed., I. D. Howarth, ASP Conf. Ser., 131, 258 Puls, J. 1991, A\&A, 248, 581

Rybicki, G. B., \& Hummer, D. G. 1991, A\&A, 245, 171

Škoda, P. 1995, Ph.D. Thesis, AsÚ AV ČR Ondřejov

Werner, K. 1989, A\&A, 226, 265

Williams, R. E. 1967, ApJ, 147, 556 